\title{
Bat Algorithm Based Beamformer for Interference Suppression by Controlling the Complex Weight
}

\author{
Tong Van Luyen ${ }^{1}$, Truong Vu Bang Giang ${ }^{2}$ \\ ${ }^{1}$ Faculty of Electronic Engineering, Hanoi University of Industry, Hanoi, Vietnam \\ 2 Vietnam National University, Hanoi, Vietnam
}

Correspondence: Truong Vu Bang Giang, giangtvb@vnu.edu.vn

Communication: received 28 April 2017, revised 17 August 2017, accepted 2 October 2017

Online publication: 7 March 2018, Digital Object Identifier: 10.21553/rev-jec.159

The associate editor coordinating the review of this article and recommending it for publication was Dr. Ha Hoang Kha.

\begin{abstract}
This study has proposed an adaptive beamformer for pattern nulling of Uniformly Spaced Linear Array (ULA) antennas for interference suppression. This beamformer has been developed based on controlling the complex weight (both the phase and the amplitude) of each array element as pattern nulling and Bat algorithm (BA) as a global optimization approach. So as to verify the proposal, a number of scenarios of ULA pattern with pre-set nulls have been conducted and compared with those of accelerated particle swarm optimization (APSO). As a result, the proposed beamformer has demonstrated the capability to place precisely single, multiple, and broad nulls at arbitrary interference directions, suppress sidelobes, and maintain a predefined main lobe. Moreover, the beamformer shows faster convergence and higher efficiency regarding null steering in pattern synthesis, as compared with an APSO-based beamformer.
\end{abstract}

Keywords- Beamformer, Bat algorithm, pattern nulling, interference suppression, ULA antennas.

\section{INTRODUCTION}

The popularity of adaptive beamformers in communication systems thank to its significant aid in enhancing performance by maximizing the effectiveness of radio spectrum usage, suppressing interference, and conserving energy. Beamformers have the ability to yield proper weights so that smart array antennas can obtain the desired pattern [1] for pattern nulling.

A few nulling techniques, namely the position-only control, the amplitude-only control, the phase-only control, and the complex weight control (including both the amplitude and the phase) have appeared in numerous studies and implementations [1,2]. Each of these methods, nonetheless, has its own pros and cons.

Among those, the position-only control [3] is complicated and difficult in terms of accuracy control, because of the need to use a mechanical driving system. The amplitude-only control [4], in contrast, is an effortless technique because it only makes adjustments in the amplitude excited at each element. Our previous research [5] adopted this control, proposed and conducted successfully BA-based beamformer for adaptive steering nulls of ULA antennas pattern. Still, $[4,5]$ has a limitation, which is the simultaneous and symmetrical placing of the nulling points on two opposite sides of the main beam. Phase-only pattern nulling is simpler and more suitable for phased arrays, considering the fact that the necessary controls are available without further expense. Moreover, [6-8] shows that steering the direction of the main beam is simple by adjustments of the phase weights. In our previous study [9], a BAbased beamformer, which is used for ULA antennas pattern nulling by phase-only control, has been proposed and successfully implemented. Still, the inherent nonlinearity of this control is obvious, and the nulling equations can be linear only with the assumption of small phase perturbations [8].

Due to the need for a controller, a phase shifter, and an attenuator for each array element, the complex weights method has the highest level of complexity and the highest cost. Yet, at the same time, its efficiency and flexibility are also the most impressive [8, 10-12].

Recently, in order to overcome limitations of the classical optimization techniques, which are the issues of getting stuck in local minima in some conditions and inflexibility, various nature-inspired optimization algorithms proceeding from computational intelligence methods have been developed. These algorithms such as genetic algorithm (GA), ant colony optimization, particle swarm optimization (PSO), differential evolution, bee algorithm, and clonal selection have been used and demonstrated as having better performance and flexibility than the classical techniques [3-7, 13-16]. The pros and cons of these algorithms have been shown when they are applied in the array pattern synthesis. In addition, GA and PSO have had a large-scale implementation regarding synthesizing array patterns $[2,13-$ 15]. PSO has been proven to be much faster and more efficient than GA $[5,9,16]$. There are several versions of PSO that have been proposed. Among those, in terms of improved convergence speed and simplicity, acceler- 
ated particle swarm optimization (APSO) developed by Xin-She Yang has been considered as a good one [17, 18] for reference.

BA has been considered a new optimization algorithm that takes inspiration from the natural world the bat's practice of using echolocation to forage for food, detect obstacles, and find their way into roosts at night. The successful application of this algorithm has solved various engineering issues [17, 19]. It has been proved that BA is better than PSO and GA with regards to convergence, robustness and precision [19]. This algorithm was first applied for adaptive beamforming in [20]. As for running time, BA has been shown to be a highly potential optimization method for adaptive beamforming [20]. Still, this study was in initial stage, hence, it did not sufficiently analyze the use of BA in beamforming.

This study proposes a BA-based beamformer to synthesize ULA antennas pattern with arbitrary nullsteering capacities. Our proposal sets the controlling parameter as the complex weight (both the phase and the amplitude) of excitation for each array element, with the ultimate goal as array pattern synthesis with nulls at directions of interference. Five scenarios have verified the proposed beamformer and put it in comparison with an APSO-based one. It has been shown in the result that the beamformer demonstrates high competency at null steering towards to directions of interference, suppressing side lobes, with better efficiency than APSO-based beamformer.

\section{Problem Formulation}

In our study, an ULA antenna of even number of isotropic elements $2 N$ ( $N$ is an integer) has been used and shown in Figure 1. The array elements are positioned along the $x$-axis, and are symmetric around the array's center. The definition of the array factor is as follows:

$$
A F(\theta)=\sum_{n=-N}^{N} \omega_{n} e^{j n d k \sin (\theta)},
$$

where $\omega_{n}=a_{n} e^{j \delta_{n}},\{n=(-N, \ldots,-2,-1,1,2, \ldots, N)\}$, is the complex weight excited to $n^{\text {th }}$ element; $k=\frac{2 \pi}{\lambda}$ is the wave number; $\lambda$ is wave length; $d$ is the element spacing.

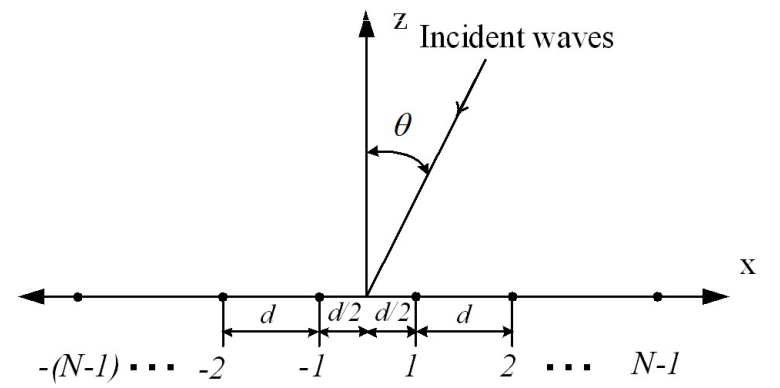

Figure 1: Geometry of ULA antennas of $2 \mathrm{~N}$ elements.
In order to obtain better effectiveness of pattern nulling, the minimum phase perturbation requires odd phase shift [21] $\left(\delta_{-n}=-\delta_{n}\right)$. By applying this, antisymmetrical pattern around the main beam direction $(\theta=0)$ is obtained as well. The amplitude has been chosen as an even function which is symmetric around the center of the array. Using the selected odd symmetric phase shift and even symmetric amplitude, the number of weight controllers and attenuators will be reduced by half.

Since $a_{-n}=a_{n}$,

$$
\sin \left(-n d k \sin (\theta)+\delta_{-n}\right)=-\sin \left(n d k \sin (\theta)+\delta_{n}\right),
$$

and

$$
\cos \left(-n d k \sin (\theta)+\delta_{-n}\right)=\cos \left(n d k \sin (\theta)+\delta_{n}\right),
$$

the array factor in (1) can be rewritten as:

$$
A F(\theta)=2 \sum_{n=1}^{N} a_{n} \cos \left(n d k \sin (\theta)+\delta_{n}\right) .
$$

The objective function $F$ has been built from $[5,9]$ :

$$
F=\left\{\begin{array}{c}
10000 \sum_{i=1}^{I}\left|A F_{0}\left(\theta_{i}\right)\right|^{2}, \text { for } \theta=\theta_{i} \\
\sum_{\theta=-90^{\circ}}^{90^{\circ}}\left|A F_{0}(\theta)-A F_{d}(\theta)\right|^{2}, \theta \neq \theta_{i}
\end{array}\right.
$$

where (3.1) is used to place null points, in which $I$ is the maximum number of interferences; (3.2) is used for reduction of SLL and for maintaining the main beam; $A F_{0}$ and $A F_{d}$ are respectively the optimized array factor which is achieved by employing an optimization algorithm (BA in this paper), and the desired array factor; $\theta_{i}$ are angles of null points.

\section{Proposal of the Beamformer}

\subsection{BAT Algorithm}

BAT algorithm (BA) is a novel metaheuristic algorithm for global optimization initiated by Xin-She Yang in 2010 [19]. It is inspired by the natural behaviors of microbats in terms of using the echolocation of ultrasonic waves to sense distance.

In BA $[17,19]$, the new locations $\left(x^{i+1}\right)$ and new velocities $\left(v^{i+1}\right)$ of bat $(i)$ at time step $t$ are decided by:

$$
\begin{aligned}
f_{i} & =f_{\min }+\left(f_{\max }-f_{\min }\right) \beta, \\
v_{i}^{t+1} & =v_{i}^{t}+\left(x_{i}^{t}-x_{\text {cbest }}\right) f_{i}, \\
x_{i}^{t+1} & =x_{i}^{t}+v_{i}^{t+1},
\end{aligned}
$$

where: $f_{i}$ is the frequency of ultrasonic waves; $\beta \in[0 ; 1]$ is an arbitrary vector built from a uniform distribution; $x_{\text {cbest }}$ represents the current global best solution that is determined by the comparison of the solutions from $n$ bats; Frequency range is defined by $f_{\min }$ and $f_{\max }$, which are selected according to the domain size of the problem of concern.

At the beginning, each bat is imposed with arbitrary frequency uniformly drawn from $\left[f_{\min } ; f_{\max }\right]$. As for local search, when a solution is picked from the current 
best solutions, a new one is made locally using random walk as:

$$
x_{\text {new }}=x_{\text {cbest }}+\varepsilon A^{t},
$$

where $\varepsilon \in[0,1]$ is a random number; $A^{t}$ is the average loudness of all the bats at time step $t$.

Moreover, in consecutive iterations, the $A_{i}$ and the rate $r_{i}$ of emission pulse can be renewed by

$$
\begin{aligned}
A_{i}^{t+1} & =\alpha A_{i}^{t}, \\
r_{i}^{t+1} & =r_{i}^{0}[1-\exp (-\gamma t)],
\end{aligned}
$$

in which $0<\alpha<1$ and $0<\gamma$ are constants.

\subsection{Proposed Beamformer}

A BA-based beamformer using complex weight control for interference suppression has been developed from $[5,9,22]$. Figure 2 is the presentation of its flowchart. Below is the description of how the beamformer operates:

Initializing (I):

- Establishing the input data, namely: number of array elements $(N)$, Direction of Arrival (DOA) of Interferences; number of iteration (i); and the termination criterion, for example, the maximum number of iterations $\left(\operatorname{Max}_{I}\right)$ or the desired value of objective function (Threshold).

- Initializing bat population with parameters of each bat follows: location $x_{i}$; velocity $v_{i}$; pulse frequency $f_{i}$; pulse rate $r_{i}$; and loudness $A_{i}$. Each bat is in corresponds with a potential solution.

Finding the best solution $(F)$ :

The beamformer consecutively defines current best solutions based on the BA. The operation is completed when the termination criterion is satisfied. After that, the last current best solution is the final best one.

Building the weight vector of arrays $(B)$ :

The beamformer determines the corresponding weight vector of ULA antenna based on the best solution. This weight vector will be used for ULA pattern nulling.

\section{Numerical Results}

Five scenarios are going to be carried out to illustrate the performance of the proposal. The fact that Chebyshev array weights distribution generates the best pattern in regard to a trade-off between the side lobe level and the first null beamwidth for uniformly spaced arrays [23]. Thus, this study has selected the array factor of Chebyshev array ( $-30 \mathrm{~dB}$ SLL, $\lambda / 2$ inter-element spacing, and 20 isotropic elements) to control SLL and the beamwidth of the main beam. Equation (3) has theta angle with one-degree step size.

In all scenarios, the parameters for BA have been selected as: population size (pop): 500 and number of iterations (emphite): 100 (with the exception of the first scenario); random walk has step size as 0.01 ; boundary

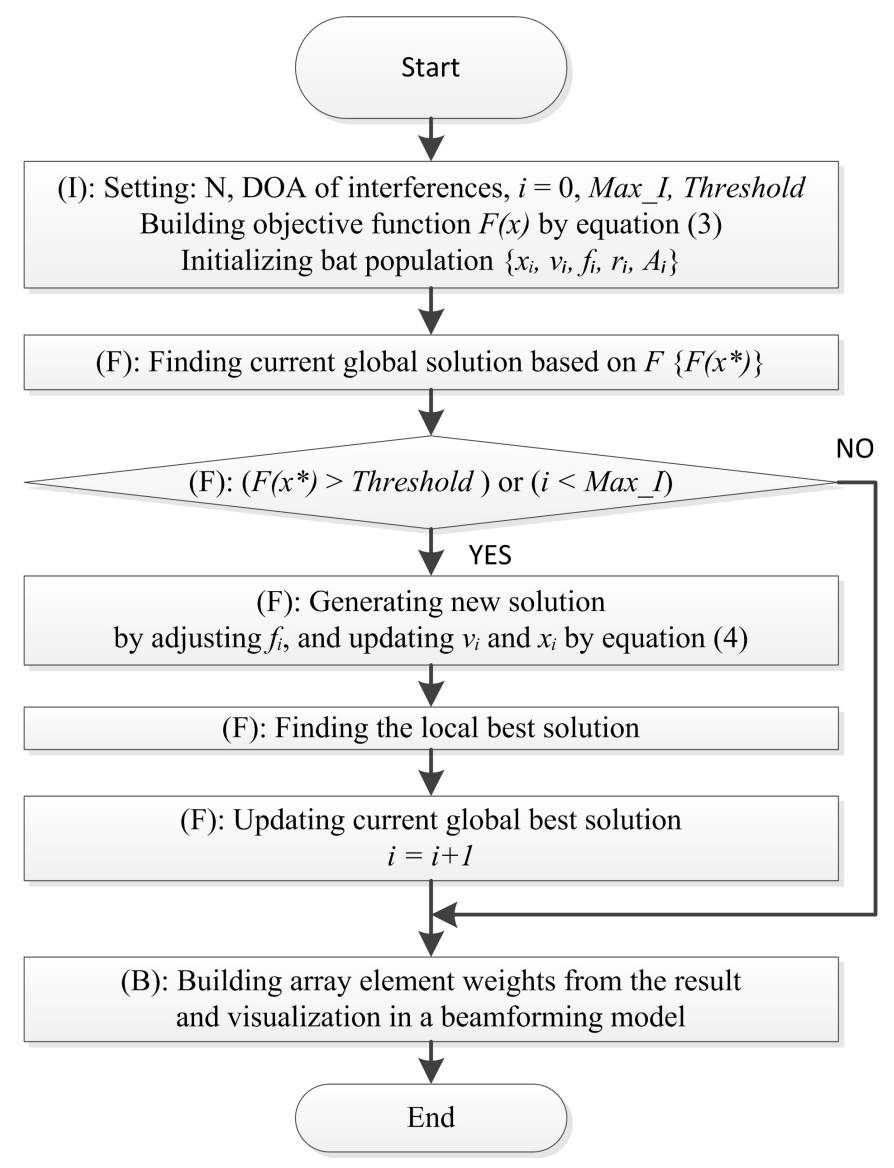

Figure 2: Flowchart of the proposed beamformer.

frequency values: $f_{\min }=0$ and $f_{\max }=1$. The acceleration parameters of APSO have been chosen as $\alpha=0.2$ and $\beta=0.5$ [18].

In [2], Randy L Haupt discovered that the phase exerted a bigger impact on the main beam than the amplitude, and adaptive nulling with insignificant phase disruption would not lead to considerable degradation to the main beam. In addition, the size of the range of variable phase weight is inversely proportional to the speed of convergence. Therefore, for all investigated scenarios of pattern nulling, search value $\left(x_{i}\right)$ has been chosen as: (1) variable phase of weight in the range of -0.1 to 0.1 radian, and (2) variable amplitude of weight in the range of 0 to 1 .

The first scenario is the initial stage to assess how the proposed beamformer is operated (See 4.1). The second to the fifth scenario are used for the purposes of investigating and comparing the capability of nullsteering of the proposed beamformer (See 4.2-4.4) with that of the APSO-based beamformer. Consequently, all simulation results presented in figures are averaged values of 50 Monte Carlo simulations.

\subsection{Convergence Characteristics}

In the first scenario, the convergence capability of our proposed beamformer, which is used in the case of gaining the desired optimization pattern as Chebyshev array pattern with $-30 \mathrm{~dB}$ SLL, has been investigated. An initial population has been created randomly; and 


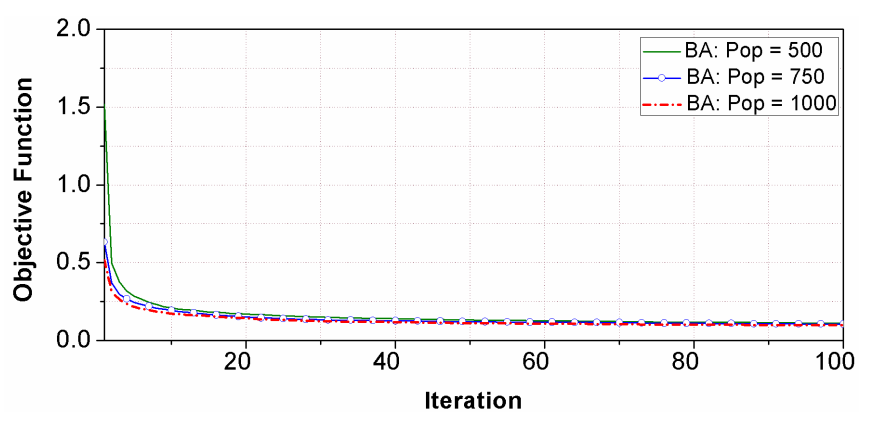

Figure 3: Objective function of BA with different population sizes.

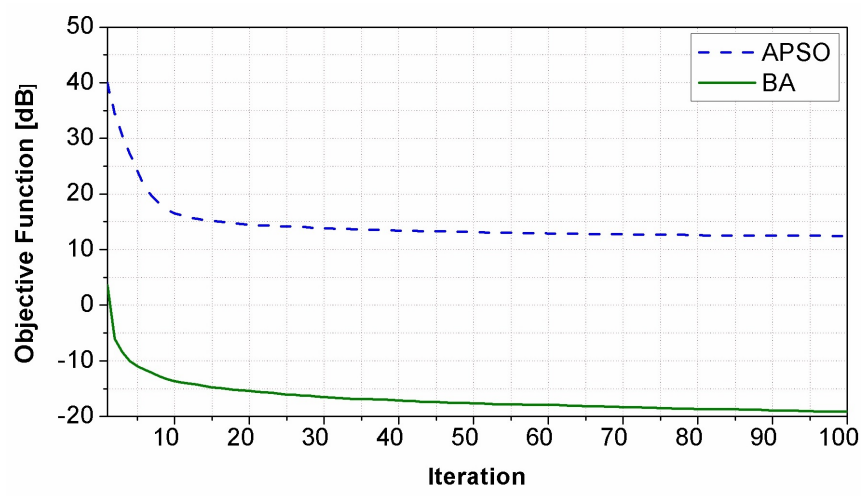

Figure 4: Objective function between BA and APSO.

the population size alternates as 500, 750, and 1000 with 100 iterations. Figure 3 exhibits the result, which shows that the convergence speed of the beamformer is impressive. The population size is inversely proportional to the number of iterations required. When population size reaches 1000, only 2 iterations are needed to achieve $F<0.5(F \mathrm{~dB}<-6 \mathrm{~dB})$.

Furthermore, to illustrate the efficiency of the proposed beamformer, its convergence rate will be compared with the APSO-based one. Their convergence rates with population of 500 and 100 iterations have been assessed for sidelobe suppression and illustrated in Figure 4. It appears that the BA-based beamformer has a much higher speed of convergence than that of the APSO-based one.

\subsection{Pattern with Single Null}

The second scenario demonstrates the optimized pattern with a single null. This null can be set at an arbitrary angle, which is selected at the peak of the second sidelobe $\left(14^{\circ}\right)$ in this case. One bat (the location) in the population is initialized by weights of Chebyshev array with SLL of $-30 \mathrm{~dB}$ while the rest are random.

Figure 5 presents optimized patterns with a single null achieved by our proposal based on BA, and APSO, in which population size is 500, and number of iterations is 100 . The BA pattern retains most of characteristics of the initial Chebyshev pattern like half power beamwidth $\left(\mathrm{HPBW}=7.64^{\circ}\right)$ and SLL $(-30 \mathrm{~dB})$ with the exception of a few side lobes with maximum SLL of $-27.02 \mathrm{~dB}$ and null point at $14^{\circ}$. The null depth level (NDL) is $-86.19 \mathrm{~dB}$. In addition, the single null pattern optimized by the BA is better than that of the APSO regarding NDL at the desired null point.

\subsection{Pattern with Multiple Nulls}

In the third scenario, the beamformer will be used to separately set multiple nulls at $-33^{\circ},-26^{\circ},-14^{\circ}$, $-40^{\circ}, 20^{\circ}$, and $40^{\circ}$, which correspond to the peaks of six sidelobes next to the main beam of Chebyshev array pattern. As shown in Figures 6, 7, the patterns with multiple nulls at the predefined locations have been exactly obtained. All the NDLs are deeper than $-74 \mathrm{~dB}$, all the SLLs are lower than $-24 \mathrm{~dB}$ (Figure 6) or $-27 \mathrm{~dB}$ (Figure 7), and HPBW approximately equals to that of Chebyshev pattern. The BA pattern shows advantages over the APSO pattern in terms of NDL.

\subsection{Pattern with Broad Null}

The requirement of a broad null is inevitable in interference suppression applications, providing that DOA of interferences do not change significantly through time, or they are not correctly identified, or a null is constantly steered to gain an appropriate signal to noise ratio. In the fourth scenario, the patterns with broad nulls placed at the target sectors of $\left(\left[-50^{\circ},-20^{\circ}\right]\right)$ or $\left(\left[-30^{\circ},-20^{\circ}\right]\right.$ and $\left.\left[45^{\circ}, 60^{\circ}\right]\right)$ have been obtained and illustrated in Figure 8 and 9 as a demonstration of the capability of broad interference suppression. It can be observed that a broad null (minimum NDL $<-55 \mathrm{~dB}$ ) on the BA patterns at that target sector has been obtained. The beamwidth stays the same without significant changes, and with maximum SLL of $-18 \mathrm{~dB}$. According to the results, BA pattern surpasses the APSO one in terms of NDL.

All aforementioned scenarios have set precisely the null points of the patterns, and maintain the beamwidths. Notwithstanding this, SLLs were bigger than $-30 \mathrm{~dB}$. To hold maximum SLL at a predefined value $(-30 \mathrm{~dB})$ and a broad null at the target sectors of $\left[-30^{\circ},-20^{\circ}\right]$ and $\left[45^{\circ}, 60^{\circ}\right]$ as well, the fifth scenario has been conducted, in which $A F_{d}$ has been substituted by the array factor of Chebyshev array with SLL of $-44 \mathrm{~dB}$. Optimized patterns have been shown in Figure 10. From the result of the simulations, there exists a trade-off between the SLL and the beamwidth of the patterns, which possess maximum SLL of $-30 \mathrm{~dB}$ and a broadened HPBW. However, the results indicate that BA pattern gives a greater performance with respect of NDL.

As shown in all the scenarios, the SLLs have been reduced to low values however these SLLs is not completely controlled under a predefined value.

\section{Conclusions}

This study has proposed and implemented successfully a BA-based beamformer for ULA antennas pattern nulling that employs the complex weight control. Five scenarios including operation speed, pattern nulling with single, multiple, and broad nulls have verified the 


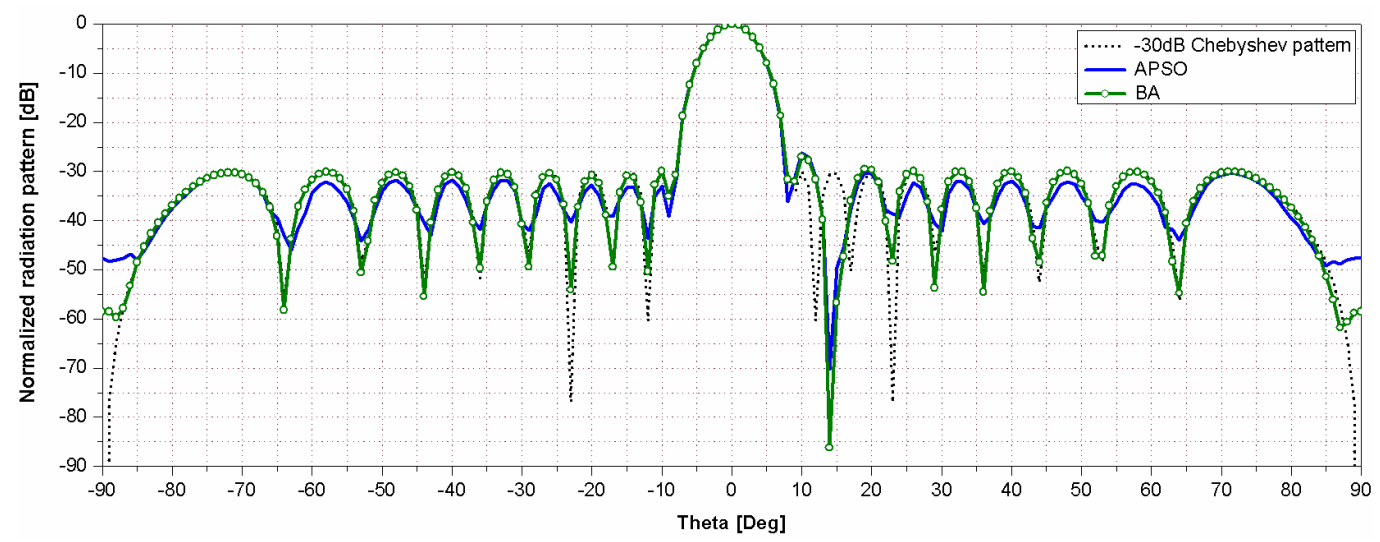

Figure 5: Optimized patterns with single null at $14^{\circ}$.

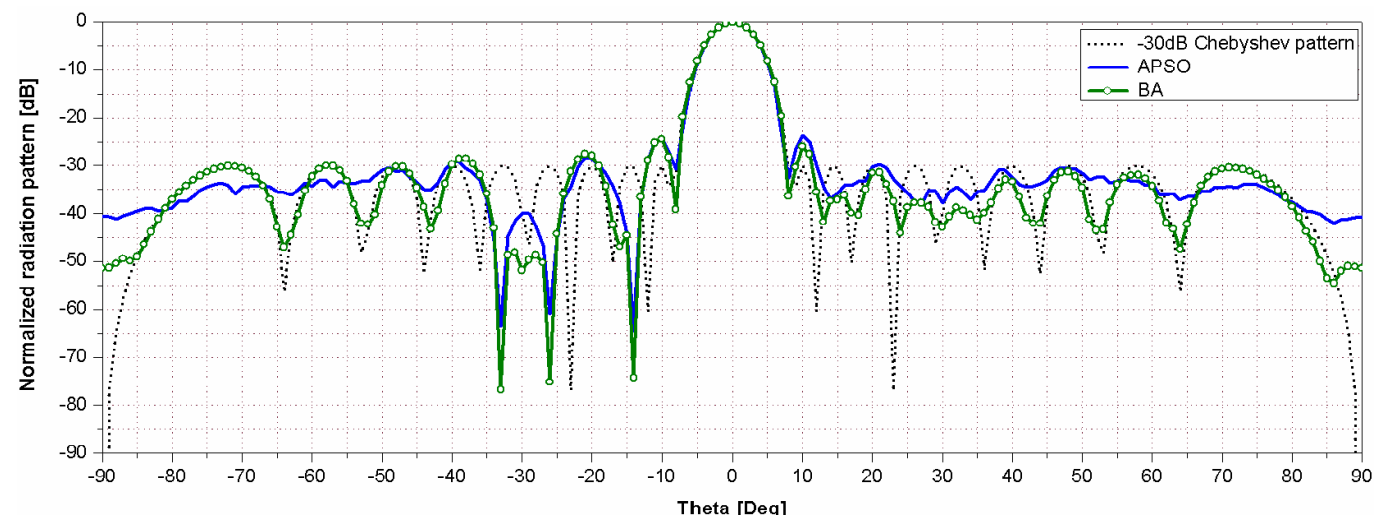

Figure 6: Optimized pattern (three nulls: $-33^{\circ},-26^{\circ}$, and $-14^{\circ}$ ).

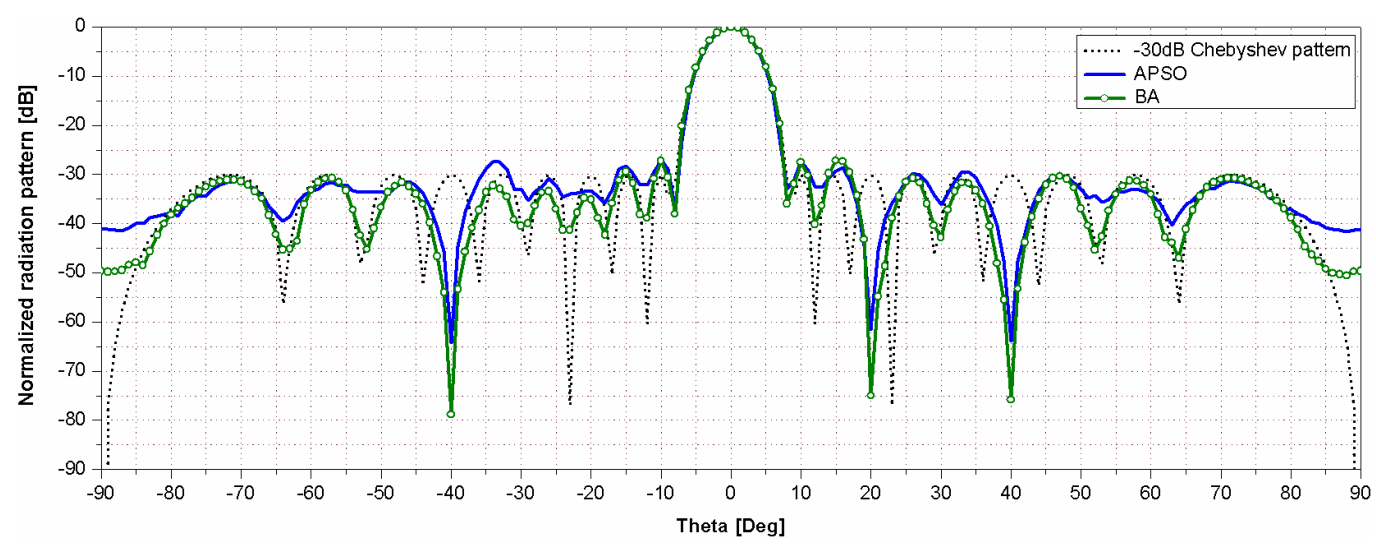

Figure 7: Optimized pattern (three nulls: $-40^{\circ}, 20^{\circ}$, and $40^{\circ}$ ).

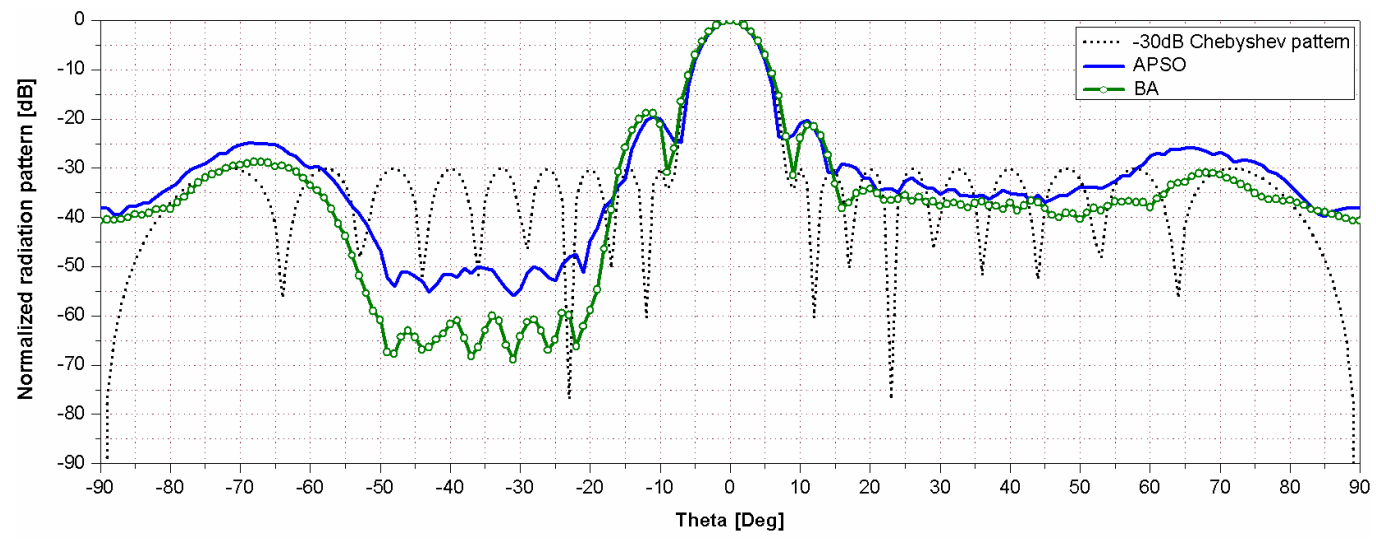

Figure 8: Optimized pattern (a broad null from $-50^{\circ}$ to $-20^{\circ}$ ). 


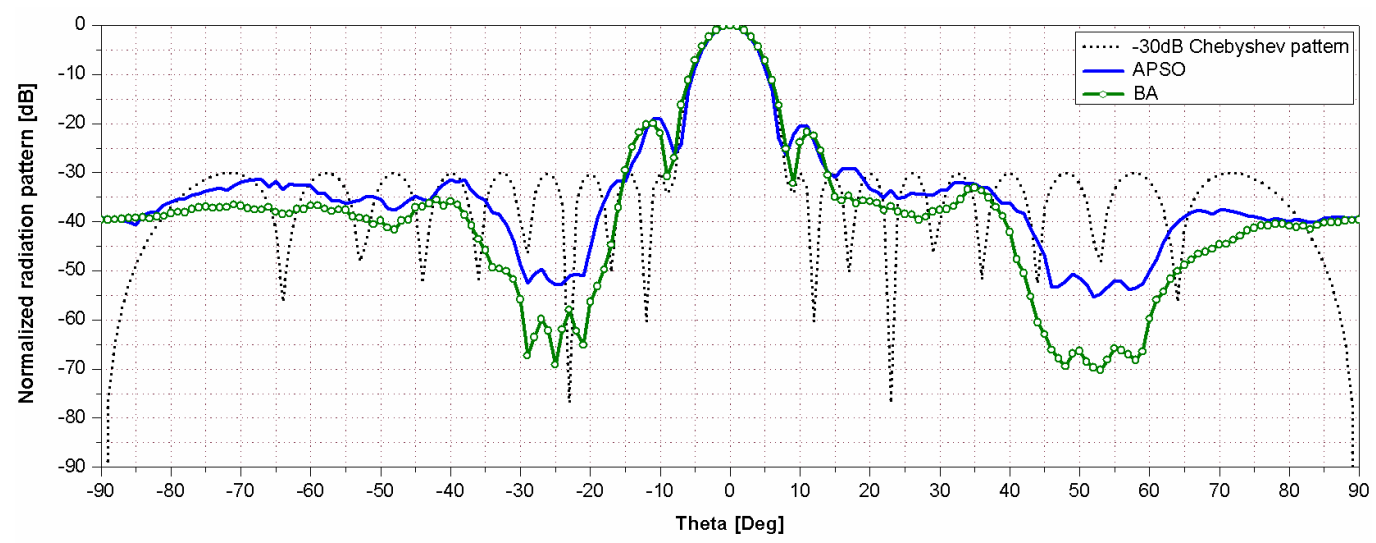

Figure 9: Optimized pattern (two broad nulls: $\left(\left[-30^{\circ},-20^{\circ}\right]\right.$ and $\left.\left.\left[45^{\circ}, 60^{\circ}\right]\right)\right)$.

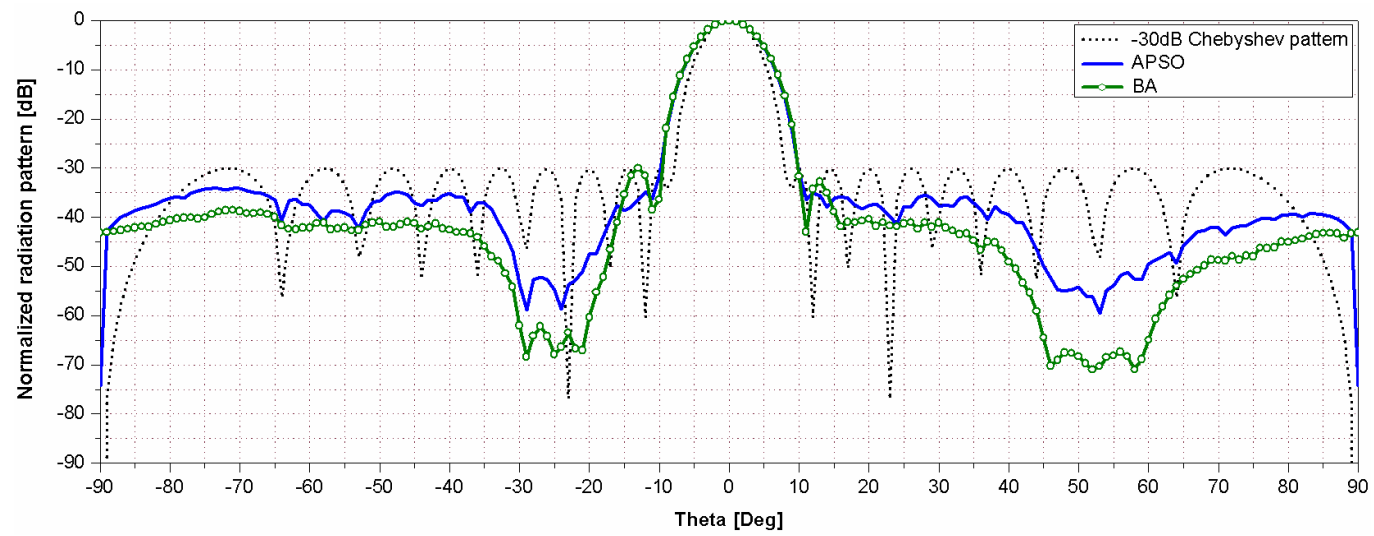

Figure 10: Optimized pattern (two broad nulls: $\left(\left[-30^{\circ},-20^{\circ}\right]\right.$ and $\left.\left[45^{\circ}, 60^{\circ}\right]\right)$ and maximum SLL of $\left.-30 \mathrm{~dB}\right)$.

pattern nulling ability of the proposal. Using the beamformer, these nulls can be imposed accurately to arbitrary directions of interferences while the patterns have maintained the main lobe and low SLL. Moreover, the proposed beamformer demonstrates more impressive competency with reference to convergence speed and adaptive pattern nulling in array pattern synthesis, as compared to APSO-based beamformer. This study has shown the verification of the proposal in the conditions of isotropic array elements and without mutual coupling. Toward realistic electromagnetic effect in relation with antennas and propagation, the effect of mutual coupling and element patterns will be investigated in

\section{ACKNOWLEDGMENT}

This work has been partly supported by Vietnam National University, Hanoi (VNU), under Project No. QG. 16.27.

\section{REFERENCES}

[1] H. L. Van Trees, Optimum Array Processing: Part IV of Detection, Estimation, and Modulation Theory. New York: Wiley \& Sons, 2002, vol. 1.

[2] R. L. Haupt, Antenna Arrays: A Computational Approach. John Wiley \& Sons, 2010.

[3] K. Guney and M. Onay, "Bees algorithm for null synthesizing of linear antenna arrays by controlling only the element positions," Neural Network World, vol. 17, no. 2, pp. 153-169, 2007.
[4] _ " "Amplitude-only pattern nulling of linear antenna arrays with the use of bees algorithm," Progress In Electromagnetics Research, vol. 70, pp. 21-36, 2007.

[5] T. V. Luyen and T. V. B. Giang, "Null-steering beamformer using bat algorithm," Journal of Applied Computational Electromagnetic Society, vol. 33, no. 1, pp. 23-29, 2017.

[6] A. Akdagli and K. Guney, "Null steering of linear antenna arrays by phase perturbations using modified tabu search algorithm," Journal of Communications Technology and Electronics, vol. 49, no. 1, pp. 37-42, 2004.

[7] M. Mouhamadou, P. Vaudon, and M. Rammal, "Smart antenna array patterns synthesis: Null steering and multi-user beamforming by phase control," Progress In Electromagnetics Research, vol. 60, pp. 95-106, 2006.

[8] K. Guney and M. Onay, "Bees algorithm for interference suppression of linear antenna arrays by controlling the phase-only and both the amplitude and phase," Expert systems with Applications, vol. 37, no. 4, pp. 3129-3135, 2010.

[9] T. V. Luyen and T. V. B. Giang, "Interference suppression of ULA antennas by phase-only control using bat algorithm," IEEE Antennas and Wireless Propagation Letters, vol. 16, pp. 3038-3042, 2017.

[10] D. Karaboga, K. Guney, and A. Akdagli, "Antenna array pattern nulling by controlling both amplitude and phase using modified touring ant colony optimization algorithm," International Journal of Electronics, vol. 91, no. 4, pp. 241-251, 2004.

[11] M. Mouhamadou and P. Vaudon, "Complex weight control of array pattern nulling," International Journal of RF and Microwave Computer-Aided Engineering, vol. 17, no. 3, pp. 304-310, 2007.

[12] H. Steyskal, R. Shore, and R. Haupt, "Methods for null control and their effects on the radiation pattern," IEEE 
Transactions on Antennas and Propagation, vol. 34, no. 3, pp. 404-409, 1986.

[13] D. W. Boeringer and D. H. Werner, "Particle swarm optimization versus genetic algorithms for phased array synthesis," IEEE Transactions on Antennas and Propagation, vol. 52, no. 3, pp. 771-779, 2004.

[14] K.-C. Lee and J.-Y. Jhang, "Application of particle swarm algorithm to the optimization of unequally spaced antenna arrays," Journal of Electromagnetic Waves and Applications, vol. 20, no. 14, pp. 2001-2012, 2006.

[15] M. R. R. Khan and V. Tuzlukov, "Null steering beamforming for wireless communication system using genetic algorithm," in Proceedings of the IEEE International Conference on Microwave Technology \& Computational Electromagnetics (ICMTCE). IEEE, 2011, pp. 289-292.

[16] B.-K. Yeo and Y. Lu, "Adaptive array digital beamforming using complex-coded particle swarm optimizationgenetic algorithm," in Proceedings of the Asia-Pacific Microwave Conference (APMC). IEEE, 2005, pp. 778-780.

[17] X.-S. Yang, Nature-inspired optimization algorithms. London: Elsevier, 2014.

[18] P. Florence and G. Raju, "Synthesis of linear antenna arrays using accelerated particle swarm optimization algorithm," International Journal of Computer Applications, vol. 103, no. 3, pp. 43-49, 2014.

[19] C. Cruz, J. R. González, D. A. Pelta, N. Krasnogor, and G. Terrazas, Nature Inspired Cooperative Strategies for Optimization (NICSO 2010). Springer, 2010, vol. 284.

[20] Q. Yao and Y. Lu, "Efficient beamforming using bat algorithm," in Proceedings of the IEEE MTT-S International Conference on Numerical Electromagnetic and Multiphysics Modeling and Optimization (NEMO). IEEE, 2016, pp. 1-2.

[21] R. A. Shore, "A proof of the odd-symmetry of the phases for minimum weight perturbation phase-only null synthesis," IEEE Transactions on Antennas and Propagation, vol. 32, no. 5, pp. 528-530, 1984.

[22] T. Van Luyen and T. V. B. Giang, "Design of LMS based adaptive beamformer for ULA antennas," VNU Journal of Science: Computer Science and Communication Engineering, vol. 32, no. 3, pp. 72-79, 2016.

[23] C. Dolph, "A current distribution for broadside arrays which optimizes the relationship between beam width and side-lobe level," Proceedings of the IRE, vol. 34, no. 6, pp. 335-348, 1946.

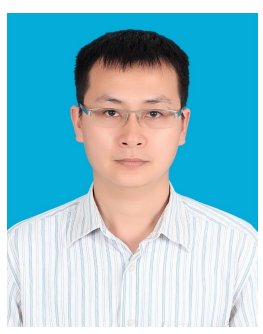

Tong Van Luyen is a lecturer at Faculty of Electronic Engineering, Hanoi University of Industry. He received the B.S. and M.S. degree from the Hanoi University of Science and Technology, in 2002 and 2004, respectively. He is now a Ph.D. student at Faculty of Electronics and Telecommunications, University of Engineering and Technology, VNU.

His research interests are in Digital Beamforming and Beamsteering for Smart Antennas, Nature Inspired Optimization.

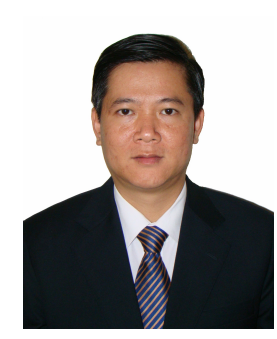

Truong Vu Bang Giang received the B.S. and M.S. degree from the VNU-University of Sciences, in 1994 and 1997, respectively, and the Dr.-Ing. (Ph.D.) degree in Electrical Engineering from the Hamburg-Harburg University of Technology, Hamburg, Germany, in collaboration with the Institute of Communications and Navigation, German Aerospcae Center, in 2006.

He is now the Deputy Director of Science and Technology Department of Vietnam National University, Hanoi and as the Secretary of the National Research Program for Sustainable Development of North-West Region of Vietnam.

He is currently the Vice President of Radio Electronics Association of Vietnam; Member of IEEE MTTs and APS. He has served as the Steering Committee (Co-Chair), Organizing Committee (Chair and Co-Chairs) or Technical Committee of ATC, REV-ECIT, VJMW, VJISAP conferences in Vietnam; Scientific and Technical Committee, International Transaction Journal of Engineering, Management, \& Applied Sciences \& Technologies (ITJEMAST).

His current research interests include Microstrip Antennas for Mobile and Handheld Devices; Analysis and Design of Conformal Antennas; Digital Beamforming and Beamsteering for Smart Antennas; Metamaterial Antennas; Design of RF Devices and Systems. 